\title{
Hematogenous CSF Blasts
}

National Cancer Institute

\section{Source}

National Cancer Institute. Hematogenous CSF Blasts. NCI Thesaurus. Code C129905.

A morphologic finding indicating the presence of blasts in a sample of cerebrospinal fluid. 